\title{
Tunicamycin-induced ER stress regulates chemokine CCL5 expression and secretion via STAT3 followed by decreased transmigration of MCF-7 breast cancer cells
}

\author{
YIMIN ZHANG $^{1 *}$, SHICHONG LIAO $^{1 *}$, WEI FAN $^{1 *}$, WEN WEI $^{1}$, \\ $\mathrm{CHANGHUA} \mathrm{WANG}^{2}$ and SHENGRONG SUN ${ }^{1}$ \\ ${ }^{1}$ Department of Breast and Thyroid Surgery, Renmin Hospital, Wuhan University, Wuhan, Hubei 430060; \\ ${ }^{2}$ Department of Pathology and Pathophysiology, School of Basic Medical Sciences, \\ Wuhan University, Wuhan, Hubei 430071, P.R. China
}

Received June 11, 2014; Accepted July 17, 2014

DOI: $10.3892 /$ or.2014.3479

\begin{abstract}
Chemokine C-C motif ligand 5 (CCL5) is an important marker related to the progression of breast cancer and is upregulated in cancer cells. However, the mechanism of the overexpression of CCL5 in tumours has not yet been clarified. The present study aimed to investigate the role of endoplasmic reticulum (ER) stress in regulating CCL5 expression and its relationship with signal transducer and activator of transcription 3 (STAT3). Meanwhile, the effect of tunicamycin, a classical ER stress inducer, and CCL5 on the transmigration of human breast cancer MCF-7 cells was observed and analysed. Compared with the normal breast epithelial tissues, expression levels of CCL5, STAT3 and CHOP, an indicator of ER stress, were significantly upregulated in breast cancer tissues. In human breast cancer MCF-7 cells, ER stress activator tunicamycin increased the expression of CCL5, STAT3 and CHOP in a time- and concentration-dependent manner. Moreover, tunicamycin-induced CCL5 expression was positively related to upregulation of unphosphorylated STAT3 (U-STAT3) but negatively related to STAT3 phosphorylation at the Tyr705 site. Furthermore, ER stress inhibited CCL5 secretion and transmigration of MCF-7 cells. This study also showed that extracellular rhCCL5 induced transmigration of MCF-7 cells which was partially blocked by the CCR5 monoclonal antibody, while knockdown of endogenous expression of CCL5 did not affect the transmigration of the cells. In conclusion, ER stress induced endogenous expression of CCL5 via elevating
\end{abstract}

Correspondence to: Professor Shengrong Sun, Department of Breast and Thyroid Surgery, Renmin Hospital, Wuhan University, 238 Jiefang Road, Wuhan, Hubei 430060, P.R. China

E-mail: sun137@sina.com

\section{*Contributed equally}

Key words: endoplasmic reticulum stress, chemokine C-C motif ligand 5, signal transducer and activator of transcription 3, transmigration, breast cancer
U-STAT3 expression; however, ER stress inhibited CCL5 secretion, which in turn, decreased the transmigration of breast cancer MCF-7 cells.

\section{Introduction}

Chemokine C-C motif ligand 5 (CCL5), also called regulated on activation normal T cell expressed and secreted (RANTES), was originally identified as a product of activated T cells and is capable of recruiting $T$ cells to inflammatory sites $(1,2)$. Further study found that CCL5 can be secreted by a variety of cells including endothelial cells, mesenchymal stem cells, tissue resident stem cells and breast cancer cells (3-6). Expression of CCL5 is upregulated in breast cancer, and is correlated to disease progression $(7,8)$. Elevated CCL5 in tumours promotes cell motility, migration, invasion and metastasis $(9,10)$ and inhibition of tumour-derived CCL5 attenuates the metastasis of tumours (11).

While the role of CCL5 in tumour progression has been recognized and investigated, the reason why CCL5 is upregulated in tumours has not been clarified yet. Research suggests that expression of CCL5 can be upregulated by signal transducer and activator of transcription 3 (STAT3) with IL-6 stimulation $(12,13)$. STAT proteins are one of several different families of latent cytoplasmic transcription factors (14). Seven members of the STAT family are found in mammals: STAT1-6 and STAT5A. When activated, STAT family members translocate to the cell nucleus acting as transcription activators (15). Two phosphorylation sites are found on STAT3 protein: Tyr705 and Ser727. An increase in the concentration of endogenous unphosphorylated STAT3 (U-STAT3) following long-term treatment with IL-6 allows U-STAT3 to compete effectively with $\mathrm{I} \kappa \mathrm{B}$ for $\mathrm{U}-\mathrm{NF} \kappa \mathrm{B}$, to form a novel transcription factor that induces CCL5 expression (12,13). However, STAT3 regulation of CCL5 expression is related to the phosphorylation of Tyr705 site rather than Ser727 (16).

Studies suggest that endoplasmic reticulum (ER) stress affects the activation of STAT3, but controversy exists. In hepatocarcinoma and retinal endothelial cells, ER stress activates STAT3 $(17,18)$, but in hepatocyte cells ER stress 
was found to inhibit activation of STAT3 (19). In tumours, moderate ER stress response functions as an anti-apoptotic mechanism protecting intracellular homeostasis. However, if severe imbalances persist which exceed the protective capacity of the ER stress response system, a shift to a pro-apoptotic mode ensues to kill the faulty cells for the benefit of the organism as a whole (20). Many types of cancer cells display chronically elevated activity levels of protective components of ER stress (21). One of the central anti-apoptotic regulators of the ER stress response is glucose-regulated protein 78 (GRP78/BiP) (22). In contrast, CCAAT/enhancer binding protein homologous transcription factor (CHOP/GADD153) represents a critical executioner of the pro-apoptotic arm of the ER stress response (23).

The present study investigated the role of ER stress induced by tunicamycin in regulating the expression of CCL5 and its relationship with STAT3 expression and phosphorylation. The role of ER stress and CCL5 on the transmigration of breast cancer MCF-7 cells was also analysed.

\section{Materials and methods}

Clinical samples and ethics statement. The study protocol was approved by the Review Committee for the Use of Human or Animal Subjects of Wuhan University. Twelve pairs of breast cancer tissues and their related normal counterparts were obtained from patients who underwent surgery at Renmin Hospital of Wuhan University from March to November 2009. All cases included were from female patients with primary breast cancer who did not undergo chemotherapy and radiotherapy before surgery. Signed consent for inclusion into the study was obtained from all patients. The median age of the patients was 53 years (range, 34 to 68$) ; 17 \%(2 / 12)$ of the patients had ductal carcinoma in situ (DCIS), 50\% (6/12) had stage I breast cancer, $25 \%$ (3/12) had stage II breast cancer, and $8 \%(1 / 12)$ had stage III breast cancer.

Cell culture and lentiviral vector infection. The human breast cancer cell line MCF-7 was purchased from the American Type Culture Collection (ATCC, Manassas, VA, USA) and maintained in RPMI-1640 medium (Hyclone, Thermo Scientific, Logan, UT, USA), supplemented with $10 \%$ (v/v) fetal calf serum (FCS; Hyclone) and $1 \%(\mathrm{v} / \mathrm{v})$ penicillin/ streptomycin (Invitrogen, Grand Island, NY, USA) at $37^{\circ} \mathrm{C}$ in a humidified incubator with $5 \% \mathrm{CO}_{2}$.

Lentiviral vectors encoding non-target control shRNA, CCL5-specific shRNA (5'-GAGACTCCGTCACAACAA CAA-3') and the infection kit were purchased from Genechem (Shanghai, China). MCF-7 cells were infected with lentiviral vectors using the kit provided by the company. Cells were then cultured in antibiotic-free normal growth medium supplemented with $5 \mu \mathrm{g} / \mathrm{ml}$ puromycin (Invitrogen) for 4 weeks to allow stable knockdown. Cells were then collected for RT-PCR and western blotting to assess knockdown efficiency and further study.

Western blot analysis. Subconfluent cells after treatment were harvested and lysed with ice-cold RIPA lysis buffer (Sigma-Aldrich, St. Louis, MO, USA) and 1\% cocktail of protease inhibitors (Sigma-Aldrich). Aliquots of $20 \mu \mathrm{g}$ lysates were separated by SDS-PAGE and transferred to a nitrocellulose membrane. After blocking with 5\% (w/v) BSA in Tris-buffered saline at $37^{\circ} \mathrm{C}$ for $1 \mathrm{~h}$, the nitrocellulose membrane was incubated with the primary antibody at $4^{\circ} \mathrm{C}$ overnight. The following primary antibodies were used: anti-tubulin, GAPDH, STAT3, phospho-STAT3 (Tyr705), (P-STAT3), CHOP and CCL5 antibodies (Cell Signaling Technology, Danvers, MA, USA). After extensive washing, the membrane was incubated with the secondary antibody at room temperature for $1 \mathrm{~h}$ (Cell Signaling Technology). Protein bands were visualized with ECL reagent (Thermo Scientific, Rockford, IL, USA) and then were quantified by Quantity One ${ }^{\circledR}$ software.

RNA isolation and real-time RT-PCR. Total RNAs were isolated from breast cancer cells or specimens and reverse transcripted to cDNA as described previously (6). Diluted cDNAs (1:10 dilution) were used in a $25-\mu 1$ real-time PCR reaction system [cDNA $2.5 \mu \mathrm{l}$, SYBR-Green Mix (Toyobo, Japan) $12.5 \mu \mathrm{l}$, sense primer $(5 \mu \mathrm{M}) 1 \mu \mathrm{l}$, antisense primer $(5 \mu \mathrm{M}) 1 \mu \mathrm{l}$, and DEPC- $\left.\mathrm{H}_{2} \mathrm{O} 8 \mu \mathrm{l}\right]$ in triplicate for each gene. The primers used in the present study were: CCL5 sense, 5'-CGTGCCCACATCAAGGAG-3' and antisense, 5'-GGA CAAGAGCAAGCAGAAAC-3'; GAPDH sense, 5'-CCATCA CCATCTTCCAGG-3' and antisense, 5'-ATGAGTCCTTCC ACGATAC-3'. Cycle parameters were $95^{\circ} \mathrm{C}$ for $1 \mathrm{~min}$ hot start and 40 cycles of $95^{\circ} \mathrm{C}$ for $15 \mathrm{sec}, 58^{\circ} \mathrm{C}$ for $15 \mathrm{sec}$ and $72^{\circ} \mathrm{C}$ for $45 \mathrm{sec}$. Blank controls with no cDNA templates were performed to rule out contamination. The specificity of the PCR product was confirmed by melting curve analysis. At the extension stage of each cycle, the value of the threshold cycle $(\mathrm{Ct})$ was recorded. A standard curve was generated by plotting the $\mathrm{Ct}$. GAPDH expression was assessed as a housekeeping gene to standardize the expression level of the target genes. The comparative expression level of the target gene $=2^{-\Delta \Delta \mathrm{Ct}}$.

Enzyme-linked immunosorbent assay (ELISA) to quantify the secretion of CCL5. Cells were treated with $10 \mu \mathrm{g} / \mathrm{ml}$ ER stress activator tunicamycin (Sigma-Aldrich) in normal cell culture medium (cells without drug treatment were set as the control). The supernatant was collected after a 24-h treatment, and the secretion of CCL5 was determined by commercial Human CCL5/RANTES DuoSet kit (R\&D Systems, Minneapolis, $\mathrm{MN}, \mathrm{USA}$ ) following the manufacturer's instructions.

Transmigration assay. The transmigration assay was carried out using Transwell chambers $(8.0 \mu \mathrm{m}$, Millipore, Billerica, MA, USA) with polycarbonate membrane filters of $8-\mu \mathrm{m}$ pore size which were used to form dual compartments in a 24-well tissue culture plate. Cells were harvested and resuspended in serum-free medium $\left(1 \times 10^{6}\right.$ cells $\left./ \mathrm{ml}\right)$. The cell suspension $(100 \mu \mathrm{l})$ was added into the upper chamber of the well. Media with $10 \%(\mathrm{v} / \mathrm{v})$ serum or with rhCCL5 (R\&D Systems) at different concentrations $(0,0.1,1,10,100 \mathrm{ng} / \mathrm{ml})$ were loaded into the bottom well. After a 24-h incubation, the Transwell chambers were fixed in $4 \%$ formaldehyde and stained with crystal violet. The non-migrating cells were carefully removed from the upper surface (inside) of the well. Cells that had migrated to the bottom surface of the filter were counted. Nine evenly spaced fields were counted in each well using 
A

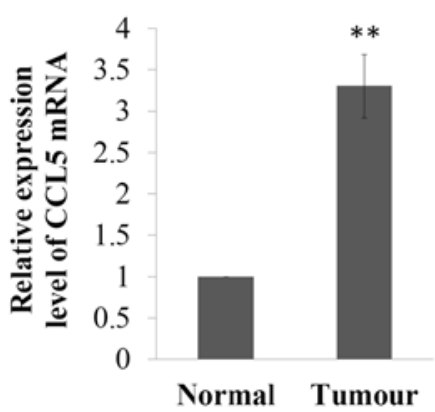

B

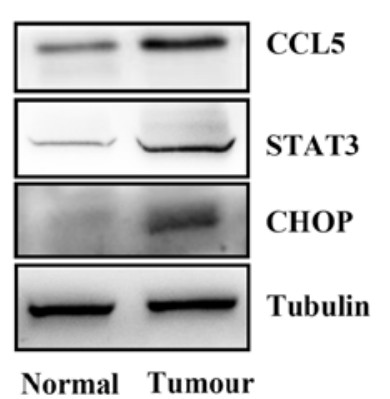

C

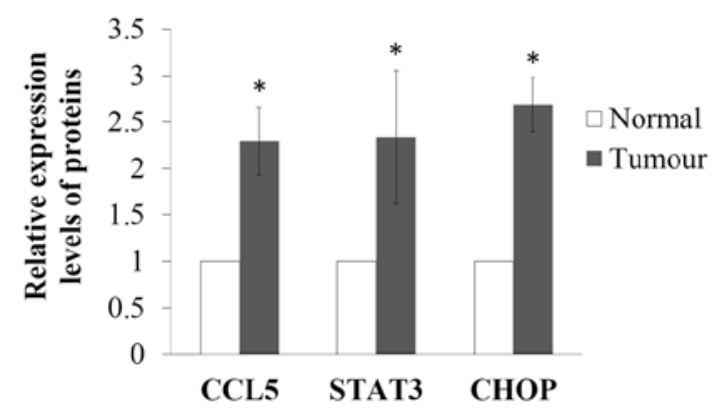

Figure 1. CCL5, STAT3 and CHOP expression in breast cancer and corresponding normal tissues. Twelve pairs of breast cancer and their corresponding normal tissues were collected. (A) Real-time RT-PCR was conducted to detect the expression of CCL5 mRNA. GAPDH was set as an internal control. The CCL5 mRNA expression in tumour tissues was presented as fold change of that in normal tissues. Data represent the mean value \pm standard deviation of 12 specimens, with each samples tested three times. ${ }^{* *} \mathrm{P}<0.01$ vs. normal tissues. (B and $\mathrm{C}$ ) Specimens were lysed and subject to western blotting to detect the expression of CCL5, STAT3 and CHOP. Tubulin was set as an internal control. (B) Representative blots for each protein. (C) Protein bands were quantified and normalized by the expression of tubulin, and expressed as n-fold change of that in the normal tissues. Data represent the mean value \pm standard deviation of 12 specimens, with each sample tested three times. ${ }^{*} \mathrm{P}<0.05$ vs. normal.

an inverted phase-contrast microscope at x20 magnification. Transmigration index $=$ number of cells counted in the test group/number of cells counted in the control group.

Statistical analysis. Data are expressed as mean \pm SD and were analysed using one-way analysis of variance (ANOVA), and the Student-Newman-Keul's test was used for individual comparisons. The statistical program SPSS 19.0 for Windows was used for analysis. A probability value $\mathrm{P}<0.05$ was considered to indicate a statistically significant result.

\section{Results}

Expression of CCL5, U-STAT3 and CHOP are upregulated in breast cancer. The CCL5 mRNA expression level in 12 pairs of breast cancer tissues and their corresponding normal breast epithelial tissues was assessed using real-time RT-PCR. As shown in Fig. 1A, CCL5 mRNA expression was significantly elevated in the breast cancer tissues, which was 3.3-fold of that in the normal breast tissues $(\mathrm{P}<0.01)$. Expression of CCL5 was also observed using western blotting, and the results revealed that CCL5 expression at the protein level was also upregulated in the tumour ( $\mathrm{P}<0.05$ vs. normal; Fig. $1 \mathrm{~B}$ and $\mathrm{C})$. In order to investigate possible mechanisms for the upregulation of CCL5 in breast cancer, the expression of STAT3, a transcriptional regulator of the CCL5 gene (12,13), and CHOP, an indicator of ER stress (23), were further assessed. Results showed that expression levels of STAT3 (2.3-fold of normal, $\mathrm{P}<0.05)$ and CHOP (2.7-fold of normal, $\mathrm{P}<0.05)$ were higher in breast cancer tissues than the levels in the corresponding normal breast epithelial tissues (Fig. 1B and C).

Tunicamycin elevates the expression of CCL5, STAT3 and CHOP in a concentration- and time-dependent manner. In order to investigate the role of ER stress in regulating expression of CCL5 and STAT3 directly, a classical ER stress activator tunicamycin was used. Breast cancer MCF-7 cells were treated with different concentrations of tunicamycin $(0$, 2.5, 5, 10 and $20 \mu \mathrm{g} / \mathrm{ml}$ ) for $24 \mathrm{~h}$. CCL5 mRNA expression was markedly elevated by tunicamycin $(\mathrm{P}<0.01$, Fig. $2 \mathrm{~A})$.
Similarly, expression of CCL5, STAT3 and CHOP were also upregulated by tunicamycin in a concentration-dependent manner (Fig. 2B). Tunicamycin $(10 \mu \mathrm{g} / \mathrm{ml})$ induced the highest CHOP and CCL5 expression levels (3.2- and 4.5-fold of the control, respectively, $\mathrm{P}<0.01$, Fig. $2 \mathrm{C}$ ). Therefore, $10 \mu \mathrm{g} / \mathrm{ml}$ was chosen as the concentration of tunicamycin used in the subsequent experiments. The effects of tunicamycin on expression of CCL5, STAT3 and CHOP after different time periods of treatment were also assessed. Western blotting showed that expression of CHOP and STAT3 started to increase after a 6-h treatment, while CCL5 expression levels were not upregulated until $12 \mathrm{~h}$ of treatment (Fig. 2D). After a 24-h treatment, expression of CCL5, STAT3 and CHOP reached the highest levels (Fig. 2E).

ER stress-induced CCL5 upregulation is positively correlated to $U$-STAT3 expression but negatively related to STAT3 phosphorylation. To confirm the role of ER stress in regulating CCL5 expression and to further investigate the mechanisms, we used ER stress inhibitor 4-PBA ( $5 \mathrm{mM}$; Sigma-Aldrich) to block the ER stress induced by tunicamycin $(10 \mu \mathrm{g} / \mathrm{ml})$ in the MCF-7 cells. As shown in Fig. 3A, CCL5 mRNA expression in the cells was induced by tunicamycin to 10.5 -fold of the control $(\mathrm{P}<0.01)$, which was blocked by 4 -PBA as the expression of CCL5 mRNA decreased to 6.1-fold of the control when cells were treated with both drugs $(\mathrm{P}<0.05$, Fig. 3A). Western blotting revealed that tunicamycin increased the expression of CCL5, STAT3 and CHOP in MCF-7 cells, compared with the control $(\mathrm{P}<0.01$, Fig. $3 \mathrm{~B}$ and $\mathrm{D})$. In contrast, when cells were treated with both tunicamycin and 4-PBA, the expression levels of these proteins were inhibited, compared with the cells treated with tunicamycin alone $(\mathrm{P}<0.01$, Fig. $3 \mathrm{~B}$ and $\mathrm{D})$. However, while STAT3 expression in breast cancer cells was upregulated by tunicamycin, the expression level of P-STAT3 (Y705) was dramatically downregulated (Fig. 3C), and the ratio of P-STAT3 (Y705) to U-STAT3 dropped to $12 \%$ of the control $(\mathrm{P}<0.01$, Fig. $3 \mathrm{E})$.

ER stress inhibits the transmigration and CCL5 secretion of $M C F-7$ cells. Since CCL5 was previously found to be involved 


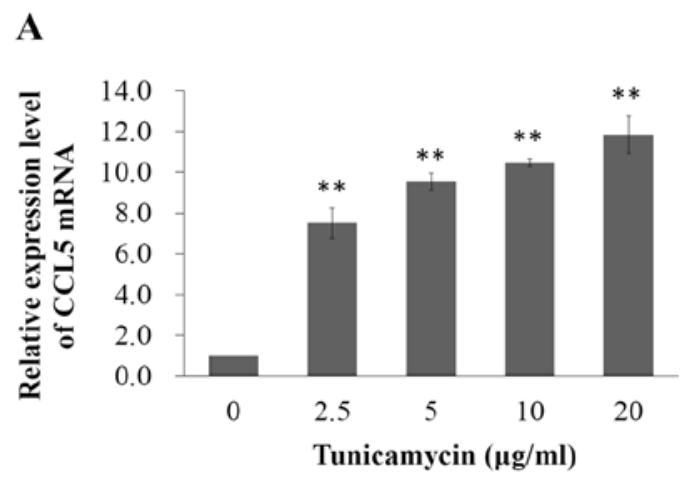

B

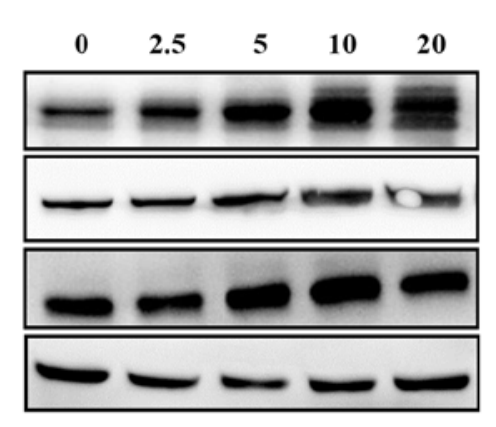

D

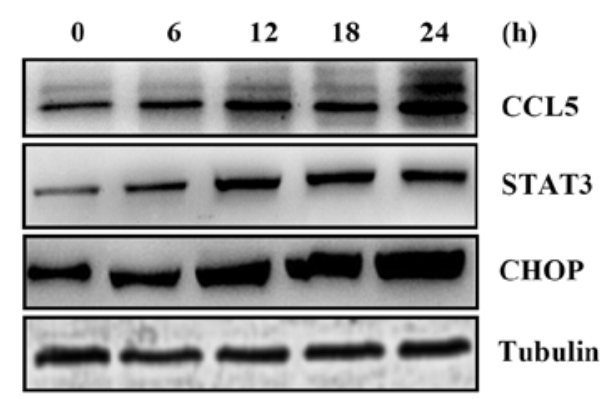

Tunicamycin

$(\mu \mathrm{g} / \mathrm{ml})$

CCL5

STAT3

CHOP

Tubulin
C

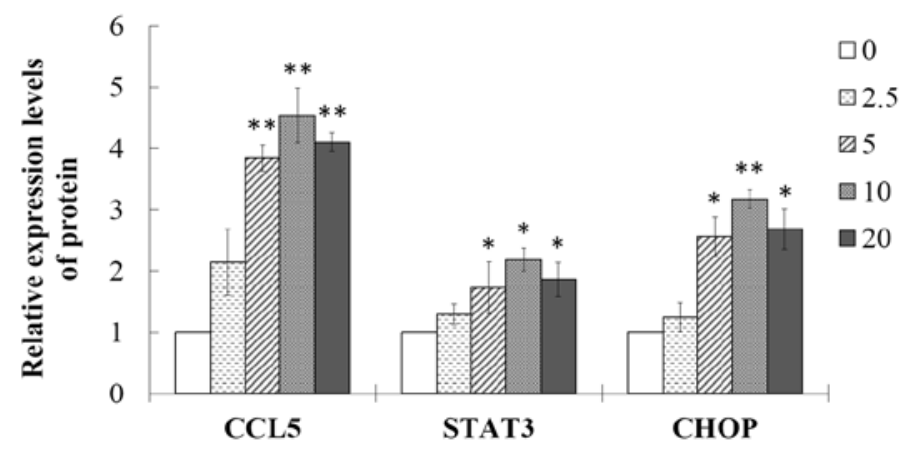

E

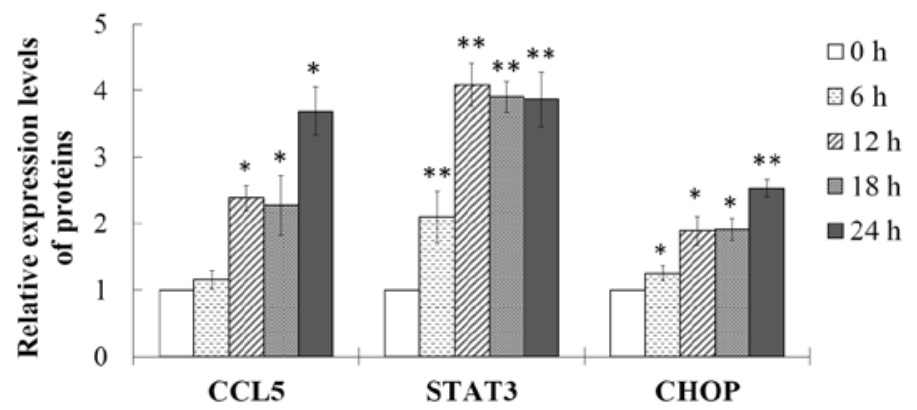

Figure 2. Effect of tunicamycin on CCL5, STAT3 and CHOP protein as well as CCL5 mRNA expression in MCF-7 cells. (A) Cultured MCF-7 cells were treated with tunicamycin at different concentrations $(2.5,5,10$ and $20 \mu \mathrm{g} / \mathrm{ml} ; 0 \mu \mathrm{g} / \mathrm{ml}$ was set as the control) for $24 \mathrm{~h}$. Real-time RT-PCR was conducted to detect the expression of CCL5 mRNA. GAPDH was set as an internal control. The CCL5 mRNA expression was presented as n-fold change of the control. Data represent the mean value \pm standard deviation of three independent experiments. ${ }^{* *} \mathrm{P}<0.01$ vs. the control. (B) Expression levels of CCL5, STAT3 and $\mathrm{CHOP}$ after treatment with tunicamycin at different concentrations (as indicated) for $24 \mathrm{~h}$ were assessed by western blotting. Tubulin was set as an internal control. (C) Relative expression levels of the proteins were quantified and presented as $\mathrm{n}$-fold change of the control. Data represent the mean value \pm standard deviation of 3 independent experiments. ${ }^{*} \mathrm{P}<0.05,{ }^{* *} \mathrm{P}<0.01$ vs. the control. (D) MCF-7 cells were treated with $10 \mu \mathrm{g} / \mathrm{ml}$ tunicamycin for $0,6,12,18$ and $24 \mathrm{~h}(0-\mathrm{h}$ treatment was set as the control), and expression levels of CCL5, STAT3 and CHOP were detected by western blotting. Tubulin was set as an internal control. (E) Relative expression levels of CCL5, STAT3 and CHOP were quantified. Data represent the mean \pm standard deviation of 3 independent experiments. ${ }^{*} \mathrm{P}<0.05,{ }^{* *} \mathrm{P}<0.01$ vs. the control.

in the migration and progression of breast cancer (6,11,24-26), we further inestigated how ER stress-induced upregulation of CCL5 affects the transmigration of MCF-7 cells. Tunicamycin inhibited the transmigration of MCF-7 to $27 \%$ of the control $(\mathrm{P}<0.01)$, which was antagonised by 4 -PBA treatment, while the transmigration index of cells treated with both tunicamycin and 4-PBA was reverted to $61 \%$ of the control $(\mathrm{P}<0.05)$, and 4-PBA treatment alone had no effect on the transmigration of MCF-7 cells (Fig. 4A). Since these results were contradictory to previous reports, which found that CCL5 induced the migration and invasion of cancer $(9,10)$, we detected the CCL5 secreted in the cell culture medium by ELISA. We found that CCL5 secretion was significantly attenuated by tunicamycin treatment to $19 \%$ of the control $(\mathrm{P}<0.01)$, while in the cells treated with both tunicamycin and 4-PBA, the secretion of CCL5 was higher than that in the tunicamycin-treated cells, which was $53 \%$ of the control $(\mathrm{P}<0.01)$. 4-PBA treatment alone did not affect CCL5 secretion of MCF-7 cells (Fig. 4B).

Extracellular CCL5 induces the transmigration of MCF-7 cells, which was partially blocked by CCR5 monoclonal antibody. Effect of extracellular CCL5 on the transmigration of 
A

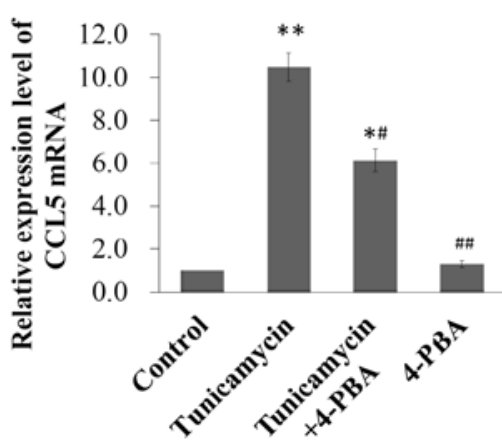

D

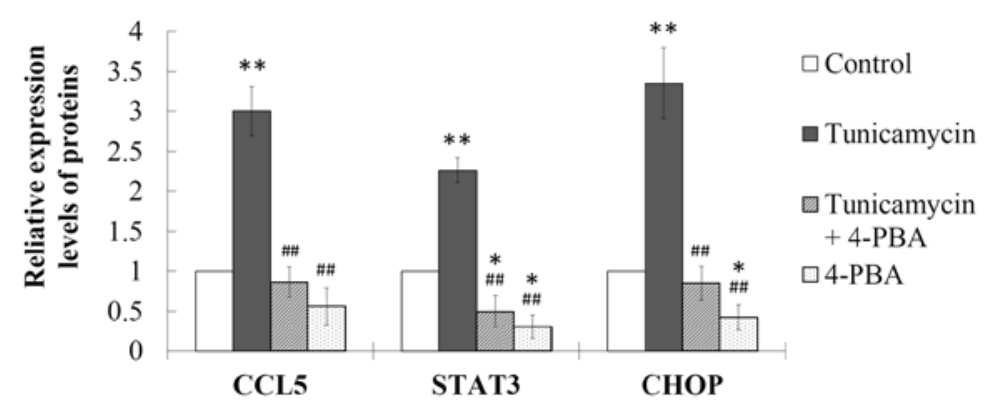

C

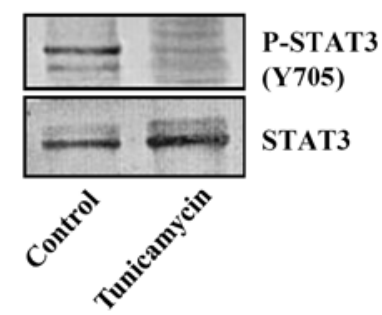

$\mathbf{E}$

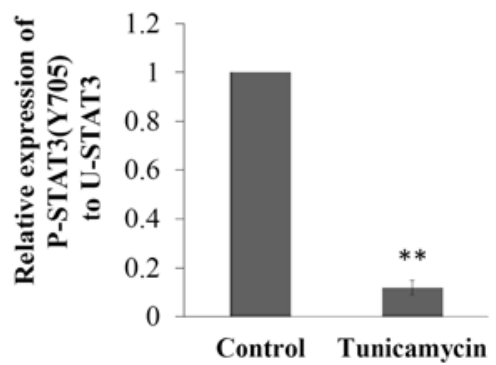

Figure 3. ER stress regulates CCL5, STAT3, P-STAT3 (Y705) and CHOP expression in MCF-7. (A) MCF-7 cells were treated with ER stress inhibitor 4-PBA $(5 \mathrm{mM})$ for $1 \mathrm{~h}$ followed by tunicamycin $(10 \mu \mathrm{g} / \mathrm{ml})$ for $24 \mathrm{~h}$ (cells with single drug treatment were set parallel; cells without drug treatment were set as the control). Expression of CCL5 mRNA was detected by real-time RT-PCR. GAPDH was set as an internal control. Data represent the mean \pm standard deviation of 3 independent experiments. ${ }^{*} \mathrm{P}<0.05,{ }^{* *} \mathrm{P}<0.01$ vs. the control and ${ }^{~} \mathrm{P}<0.05,{ }^{\# \#} \mathrm{P}<0.01$ vs. tunicamycin. (B) Expression levels of CCL5, STAT3 and CHOP were assessed by western blotting. Tubulin was set as an internal control. (D) Relative expression levels of proteins were quantified and presented as n-fold change of the control. Data represent the mean value \pm standard deviation of 3 independent experiments. ${ }^{*} \mathrm{P}<0.05,{ }^{* * *} \mathrm{P}<0.01$ vs. the control and ${ }^{\# \#} \mathrm{P}<0.01$ vs. tunicamycin. (C) Cells were treated with $10 \mu \mathrm{g} / \mathrm{ml}$ tunicamycin (cells without drug treatment were set as the control) for $24 \mathrm{~h}$ and then lysed. Expression levels of P-STAT3 (Y705) and STAT3 were assessed by western blotting. (E) The ratio of the relative expression level of P-STAT3 (Y705) to U-STAT3 was quantified. Data represent the mean \pm standard deviation of 3 independent experiments. ${ }^{* *} \mathrm{P}<0.01$ vs. the control.

A

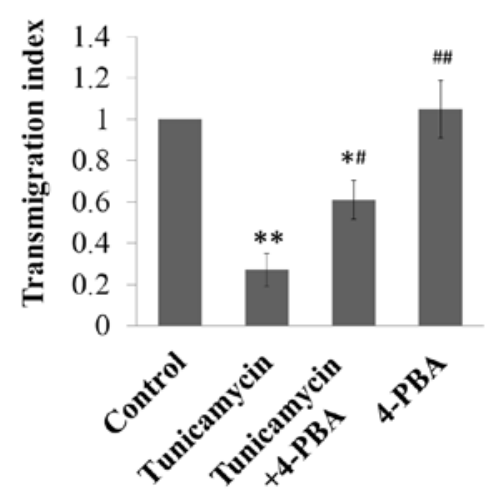

B

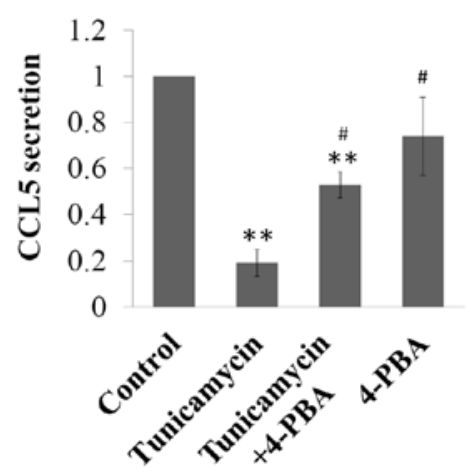

Figure 4. Effect of ER stress on the transmigration and CCL5 secretion of MCF-7 cells. MCF-7 cells were treated with 4-PBA (5 mM) for $1 \mathrm{~h}$ followed by tunicamycin $(10 \mu \mathrm{g} / \mathrm{ml})$ treatment for $24 \mathrm{~h}$ (cells with single drug treatment were set parallel; cells without drug treatment were set as the control) and (A) transmigration assay was conducted. Three independent experiments were performed in duplicate. The transmigration index was calculated and data represent the mean \pm standard deviation. ${ }^{*} \mathrm{P}<0.05,{ }^{* *} \mathrm{P}<0.01$ vs. the control and ${ }^{\#} \mathrm{P}<0.05,{ }^{\# \#} \mathrm{P}<0.01$ vs. tunicamycin. (B) Supernatants were collected for ELISA to analyse the secretion of CCL5 from cells. Three independent experiments were performed in duplicate and data represent the mean \pm standard deviation. ${ }^{* *} \mathrm{P}<0.01 \mathrm{vs}$. the control and ${ }^{\#} \mathrm{P}<0.05$ vs. tunicamycin.

MCF-7 was also observed by transmigration assay. The results showed that extracellular rhCCL5 at different concentrations induced the transmigration of MCF-7 cells, and $100 \mathrm{ng} / \mathrm{ml}$ of rhCCL5 induced the highest transmigration index in the MCF-7 cells, which was 7.9 -fold of the control $(\mathrm{P}<0.01$, Fig. 5A). Furthermore, we investigated the mechanism of the inductive effect of CCL5 on cell transmigration. The results of the transmigration assay showed that rhCCL5 induction enhanced the 
A

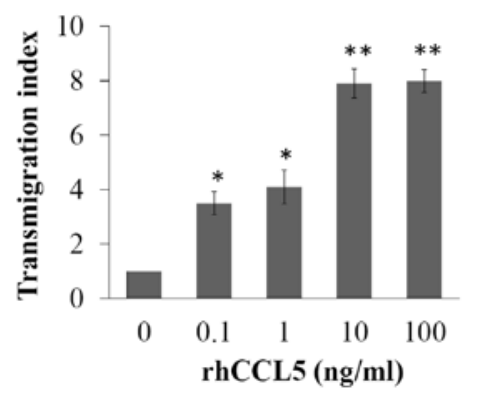

C

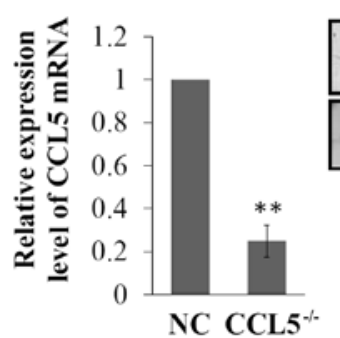

B

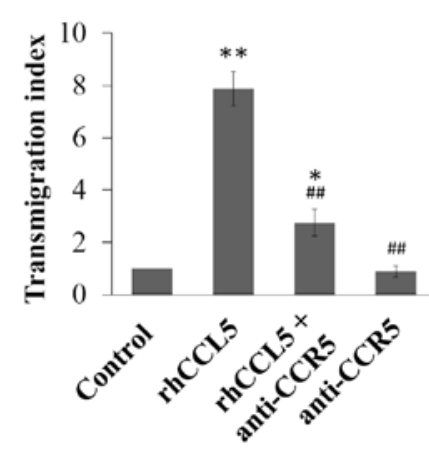

E
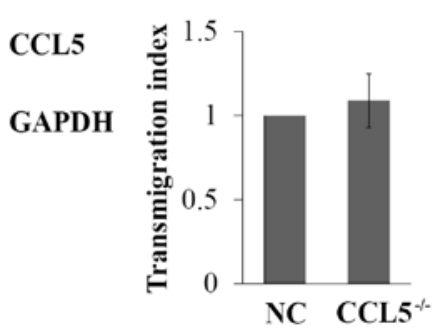

Figure 5. Role of endogenous and extracellular CCL5 in the transmigration of MCF-7 cells. (A) MCF-7 cell suspension in serum-free medium was added into the upper chamber of a Transwell system, and the lower chamber was filled with rhCCL5 at different concentrations $(0.1,1,10,100 \mathrm{ng} / \mathrm{ml}$; $0 \mathrm{ng} / \mathrm{ml}$ was set as the control) in serum-free medium, respectively. The transmigration ability of cells is shown as the transmigration index. Three independent experiments were performed in duplicate, and data represent the mean \pm standard deviation. ${ }^{*} \mathrm{P}<0.05,{ }^{* *} \mathrm{P}<0.01$ vs. the control. (B) Cell suspension in the upper well was cultured with/without $500 \mathrm{ng} / \mathrm{ml}$ of the CCR5 monoclonal antibody, and serum-free medium with/without $100 \mathrm{ng} / \mathrm{ml} \mathrm{rhCCL} 5 \mathrm{was}$ added into the bottom chamber. The transmigration ability was assessed using transmigration assay. Three independent experiments were performed in duplicate and data represent the mean \pm standard deviation. ${ }^{*} \mathrm{P}<0.05,{ }^{* *} \mathrm{P}<0.01$ vs. the control and ${ }^{\# \#} \mathrm{P}<0.01$ vs. rhCCL5. (C) CCL5-knockdown cells $\left(C C L 5{ }^{-/}\right)$and non-target control shRNA infected cells (NC) were subjected to real-time RT-PCR to observe the expression of CCL5 mRNA. GAPDH was set as an internal control. Data represent the mean \pm standard deviation of three independent experiments. ${ }^{* *} \mathrm{P}<0.01$ vs. NC. (D) Expression of CCL5 protein was also detected by western blotting. (E) Transmigration ability of CCL5 ${ }^{-/}$and NC MCF-7 cells was analysed by transmigration assay. Three independent experiments were performed in duplicate and data represent the mean \pm standard deviation.

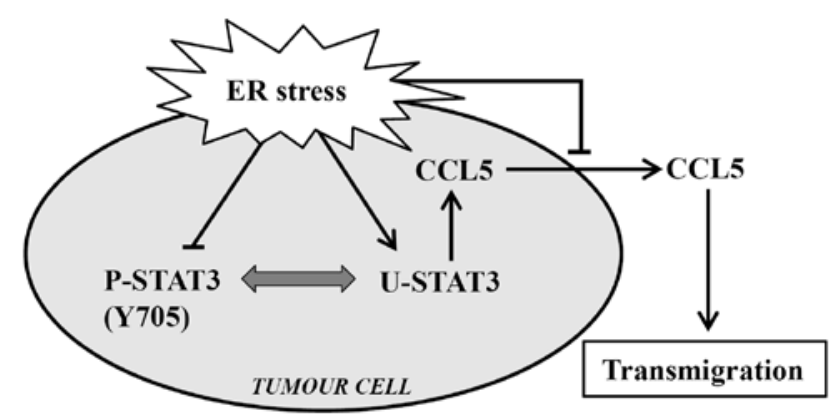

Figure 6. Diagram of the possible mechanism for ER stress-induced CCL5 overexpression and transmigration inhibition. Tunicamycin-induced ER stress inhibits STAT3 (Y705) phosphorylation and upregulates U-STAT3 expression, which in turn induces endogenous CCL5 expression. Meanwhile, ER stress inhibits the CCL5 secretion of tumour cells. Therefore, the decrease in the extracellular CCL5 level results in the decrease in the transmigration ability of cells.

transmigration ability of MCF-7 cells (7.9-fold of the control, $\mathrm{P}<0.01$ ), which was blocked by CCR5 monoclonal antibody treatment; the transmigration index decreased to $35 \%$ of the index in the rhCCL5 only group $(\mathrm{P}<0.01$, Fig. 5B). However, the transmigration index of cells treated with both rhCCL5 and CCR5 monoclonal antibody was still higher than that of the control (2.75-fold of the control, $\mathrm{P}<0.05$, Fig. 5B).
Knockdown of endogenous CCL5 expression does not affect the transmigration of $M C F-7$ cells. To investigate the role of the endogenous expression level of CCL5 on the transmigration of breast cancer MCF-7 cells, a CCL5 knockdown $\left(\mathrm{CCL}^{-/}\right) \mathrm{MCF}-7$ cell line was constructed using lentiviral vectors encoding CCL5-shRNA. The expression of CCL5 was assessed by RT-PCR and western blotting, and CCL5 expression at both the mRNA and protein levels were effectively inhibited in the $\mathrm{CCL}^{-/}$cells compared with the $\mathrm{NC}$ (Fig. 5C and D). Then the transmigration abilities of $\mathrm{NC}$ and $\mathrm{CCL}^{-/-} \mathrm{MCF}-7$ cells were assessed. No significant difference between these two cell lines was noted ( $\mathrm{P}>0.05$, Fig. 5E).

\section{Discussion}

CCL5, a classical pro-inflammatory chemokine, can be expressed and secreted by both breast cancer and non-malignant stromal cells (3-6). Although the detailed mechanisms have not yet been fully understood, the role of CCL5 in facilitating cancer progression has been recognized. However, the reason why CCL5 is upregulated in breast cancer is not well established. Studies revealed that IL-6-induced U-STAT3 upregulation in hTERT-HME1 cells led to an increase in CCL5 expression $(12,13)$. Moreover, since cancer cells display chronically elevated levels of ER stress (21) and ER stress regulates STAT3 activity (17-19), we hypothesized that 
elevated expression of CCL5 in breast cancer was attributed to ER stress-induced U-STAT3 upregulation.

In order to prove our hypothesis, we firstly assessed the expression of CCL5, STAT3 and ER stress indicator CHOP in tumour tissues from breast cancer patients and their corresponding normal breast epithelial tissues. Elevated expression of CCL5 was accompanied by upregulated expression of both CHOP and STAT3 in breast tumour tissues when compared with levels in the corresponding normal breast epithelial tissues. The results suggest that a certain correlation may exist between the expression of CCL5, STAT3 and ER stress responses of breast cancer cells. To further investigate the effect of ER stress on the expression of CCL5, we treated human breast cancer MCF-7 cells with a series of concentrations of tunicamycin, an activator of ER stress (27). Expression of CCL5 was upregulated by tunicamycin, in a concentrationand time-dependent manner. Notably, changes in STAT3 and CHOP expression in MCF-7 cells were parallel to the change in CCL5 expression after tunicamycin treatment, which is consistent with the correlation pattern of the expression of these proteins in breast cancer specimens. In order to confirm the effect of ER stress on CCL5 expression, ER stress inhibitor 4-PBA was used. Upregulation of CCL5 expression induced by tunicamycin was inhibited by ER stress inhibitor 4-PBA, and meanwhile, expression of STAT3 showed the same trend of change as that of CCL5, which supports our hypothesis that ER stress elevates endogenous CCL5 expression via STAT3 in breast cancer cells.

STAT3 regulation of CCL5 expression is related to phosphorylation of STAT3 at Tyr705 site (16). Thus, the roles of tunicamycin-induced ER stress in regulating STAT3 (Y705) phosphorylation and U-STAT3 expression were further investigated. Our results showed that the expression of P-STAT3 (Y705) was markedly decreased by tunicamycin treatment while expression of U-STAT3 was elevated. The results suggest that the upregulation of endogenous CCL5 expression in human breast cancer MCF-7 cells induced by ER stress is attributed to the elevated U-STAT3 expression, which is consistent with studies by Yang et al that demonstrated that IL-6-induced U-STAT3 upregulation in hTERT-HME1 cells led to an increase in CCL5 expression $(12,13)$.

Since ER stress upregulated CCL5 expression in breast cancer cells, and elevated CCL5 in tumours promotes cell motility, migration, invasion and metastasis $(9,10)$, tunicamycin should enhance the transmigration of cells, theoretically. Therefore, the action of tunicamycin on the transmigration of MCF-7 cells was further investigated. Importantly, our results showed that tunicamycin significantly inhibited the transmigration of MCF-7 cells. To ascertain the mechanism, we first detected CCL5 secretion (the concentration of CCL5 in the cell culture supernatants) of MCF-7 cells after tunicamycin treatment. The results revealed that although ER stress upregulated intracellular CCL5 expression, it inhibited CCL5 secretion of MCF-7 cells. According to studies of Wang et al (10) and Lin et al (28), CCL5 promotes tumour cell motility and migration by interacting with a specific receptor chemokine $\mathrm{C}-\mathrm{C}$ motif receptor 5 (CCR5). Thus, we assume that it is the extracellular CCL5 in the microenvironment of tumour cells that actually stimulates the migration, while the intracellular expression level of CCL5 does not affect the migration ability of cancer cells directly. The results confirmed the assumption further, which indicated that, on one hand, extracellular CCL5 induced the transmigration of MCF-7 cells, which was partially blocked by CCR5 monoclonal antibody; on the other hand, knockdown of endogenous CCL5 expression by shRNA did not affect the transmigration of MCF-7 cells.

Taken together, as summarized in Fig. 6, the present study suggests that ER stress induced endogenous expression of CCL5 via elevation of U-STAT3 expression, and clarifies the mechanisms of CCL5 upregulation in breast cancer. Meanwhile, ER stress inhibited CCL5 secretion, which in turn, decreased the transmigration ability of breast cancer MCF-7 cells. We determined that it was extracellular CCL5 in the microenvironment of the tumour cells that stimulated the transmigration, while the intracellular expression level of CCL5 did not affect the transmigration ability of cancer cells directly. If CCL5 is to be applied as a target to prevent the migration and metastasis of tumours, in the clinic, it may be more effective to block the CCL5-CCR5 interaction and/or to inhibit the CCL5 concentration in the microenvironment of the tumour, than to decrease the endogenous CCL5 expression in breast cancer cells. However, our findings are based on in vitro experiments, and more research, such as clinical data analysis and in vivo experiments with animal models, is required before they can be verified and applied in breast cancer treatment.

\section{Acknowledgements}

This project was supported by the Natural Science Foundation of Hubei Province, China (2011CBD489).

\section{References}

1. Schall TJ, Jongstra J, Dyer BJ, et al: A human T cell-specific molecule is a member of a new gene family. J Immunol 141: 1018-1025, 1988

2. John AE, Berlin AA and Lukacs NW: Respiratory syncytial virusinduced CCL5/RANTES contributes to exacerbation of allergic airway inflammation. Eur J Immunol 33: 1677-1685, 2003.

3. Hillyer P and Male D: Expression of chemokines on the surface of different human endothelia. Immunol Cell Biol 83: 375-382, 2005.

4. Karnoub AE, Dash AB, Vo AP, et al: Mesenchymal stem cells within tumour stroma promote breast cancer metastasis. Nature 449: 557-563, 2007.

5. Pinilla S, Alt E, Abdul Khalek FJ, et al: Tissue resident stem cells produce CCL5 under the influence of cancer cells and thereby promote breast cancer cell invasion. Cancer Lett 284: 80-85, 2009.

6. Zhang Y, Yao F, Yao X, et al: Role of CCL5 in invasion, proliferation and proportion of CD44 ${ }^{+}$CD24- phenotype of MCF-7 cells and correlation of CCL5 and CCR5 expression with breast cancer progression. Oncol Rep 21: 1113-1121, 2009.

7. Luboshits G, Shina S, Kaplan O, et al: Elevated expression of the $\mathrm{CC}$ chemokine regulated on activation, normal $\mathrm{T}$ cell expressed and secreted (RANTES) in advanced breast carcinoma. Cancer Res 59: 4681-4687, 1999.

8. Niwa Y, Akamatsu H, Niwa H, Sumi H, Ozaki Y and Abe A: Correlation of tissue and plasma RANTES levels with disease course in patients with breast or cervical cancer. Clin Cancer Res 7: 285-289, 2001.

9. Long H, Xie R, Xiang T, et al: Autocrine CCL5 signaling promotes invasion and migration of CD133(+) ovarian cancer stem-like cells via NF- $\kappa$ B-mediated MMP-9 upregulation. Stem Cells 30: 2309-2319, 2012.

10. Wang SW, Wu HH, Liu SC, et al: CCL5 and CCR5 interaction promotes cell motility in human osteosarcoma. PLoS One 7: e35101, 2012. 
11. Stormes KA, Lemken CA, Lepre JV, Marinucci MN and Kurt RA: Inhibition of metastasis by inhibition of tumor-derived CCL5. Breast Cancer Res Treat 89: 209-212, 2005.

12. Yang J, Chatterjee-Kishore M, Staugaitis SM, et al: Novel roles of unphosphorylated STAT3 in oncogenesis and transcriptional regulation. Cancer Res 65: 939-947, 2005.

13. Yang J, Liao X, Agarwal MK, Barnes L, Auron PE and Stark GR: Unphosphorylated STAT3 accumulates in response to IL-6 and activates transcription by binding to NFkappaB. Genes Dev 21: 1396-1408, 2007.

14. Bandarian V, Pattridge KA, Lennon BW, Huddler DP, Matthews RG and Ludwig ML: Domain alternation switches $\mathrm{B}(12)$-dependent methionine synthase to the activation conformation. Nat Struct Biol 9: 53-56, 2002.

15. Levy DE and Darnell JE Jr: Stats: transcriptional control and biological impact. Nat Rev Mol Cell Biol 3: 651-662, 2002.

16. Ng YP, Cheung ZH and Ip NY: STAT3 as a downstream mediator of Trk signaling and functions. J Biol Chem 281: 15636-15644, 2006.

17. Waris G, Tardif KD and Siddiqui A: Endoplasmic reticulum (ER) stress: hepatitis $\mathrm{C}$ virus induces an ER-nucleus signal transduction pathway and activates NF-kappaB and STAT-3. Biochem Pharmacol 64: 1425-1430, 2002.

18. Chen Y, Wang JJ, Li J, et al: Activating transcription factor 4 mediates hyperglycaemia-induced endothelial inflammation and retinal vascular leakage through activation of STAT3 in a mouse model of type 1 diabetes. Diabetologia 55: 2533-2545, 2012.

19. Kimura K, Yamada T, Matsumoto M, et al: Endoplasmic reticulum stress inhibits STAT3-dependent suppression of hepatic gluconeogenesis via dephosphorylation and deacetylation. Diabetes 61: 61-73, 2012.
20. Cho HY, Thomas S, Golden EB, et al: Enhanced killing of chemo-resistant breast cancer cells via controlled aggravation of ER stress. Cancer Lett 282: 87-97, 2009.

21. Schönthal AH: Endoplasmic reticulum stress and autophagy as targets for cancer therapy. Cancer Lett 275: 163-169, 2009.

22. Li J and Lee AS: Stress induction of GRP78/BiP and its role in cancer. Curr Mol Med 6: 45-54, 2006.

23. Oyadomari S and Mori M: Roles of CHOP/GADD153 in endoplasmic reticulum stress. Cell Death Differ 14: 381-389, 2004

24. Azenshtein E, Luboshits G, Shina S, et al: The CC chemokine RANTES in breast carcinoma progression: regulation of expression and potential mechanisms of promalignant activity. Cancer Res 62: 1093-1102, 2002.

25. Mañes S, Mira E, Colomer R, et al: CCR5 expression influences the progression of human breast cancer in a p53-dependent manner. J Exp Med 198: 1381-1389, 2003.

26. Yaal-Hahoshen N, Shina S, Leider-Trejo L, et al: The chemokine CCL5 as a potential prognostic factor predicting disease progression in stage II breast cancer patients. Clin Cancer Res 12: 4474-4480, 2006.

27. Ozcan U, Cao Q, Yilmaz E, et al: Endoplasmic reticulum stress links obesity, insulin action, and type 2 diabetes. Science 306: 457-461, 2004.

28. Lin S, Wan S, Sun L, et al: Chemokine C-C motif receptor 5 and $\mathrm{C}-\mathrm{C}$ motif ligand 5 promote cancer cell migration under hypoxia. Cancer Sci 103: 904-912, 2012. 